\title{
Supply and demand
}

Shaun Sellars continues his series on ethical dilemmas in dentistry which appears in every second issue of the $B D J$.

I like a guideline. Good ones have the ability to make clear the previously obscure. Take, for example, the SDCEP guidelines for management of patients at risk from MRONJ. We now have an easy to follow route to providing the best quality care for an increasing cohort of patients where before there was uncertainty.

I'm also a fan of the 2017 classification of periodontal disease and associated guidelines for treatment. Staging and grading of periodontal disease gives me, and in turn my patient, a clear insight into the progression of the disease and how it's likely to develop. One noteworthy section of this guidance is that patients with a history of periodontal disease are now to be treated as 'periodontal patients' for life. This evidence-based outcome is to be welcomed but may cause a few headaches for practitioners.

No matter what sector of dentistry we practise in, our ability to provide services to patients is limited. These restrictions could be due to time, money or any other number of factors. As a general rule, we're all working pretty much up to our limits. If we aren't then someone is doubtless trying to make us work harder and faster.

What happens then, when we tweak how one of the major diseases that we detect and treat is detected and treated? Well, initially there's a slow down while we adapt to new ways and in the case of the new periodontal guidelines, there's likely to be an increased need for ongoing maintenance care. This is fine. We're going to be providing a better quality of care to patients. But how are we going to go about doing it?

If we're already working at capacity, and there's more demand placed on our system, then something has to give. There are a few options. We could continue to devote as much time as possible to each patient, work harder and longer for the same income into the system we choose to work in. Alternatively, we could opt to see fewer patients in the same time as we have been. That probably means fewer pennies in the coffers. None of these appeal? Then we

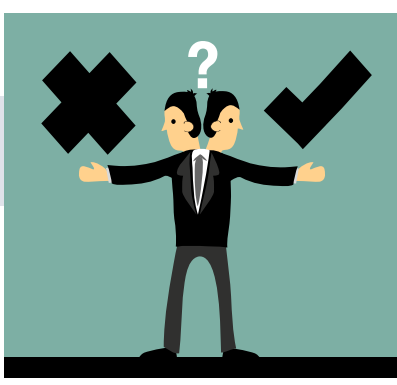

could try and squeeze the same number of patients into the same time as before. This means working more quickly and maybe cutting a few corners. Perhaps unintentionally, but perhaps not. Missing out on the occasional maintenance visit, or pushing those visits out from every three months to every six. But this may well attract some regulatory attention, and nobody wants that.

If none of these choices are particularly attractive then we're left with only one option. To change the system. The goalposts for care have moved, and that necessitates a change in how that care is delivered. Increase in demand isn't the fault of the guidelines. If we're to provide an improved service (and we should all want to do that) that service needs to be valued by those paying for it, no matter who that might be.

\section{Clean teeth linked to healthy heart}

A new study published in the European Journal of Preventive Cardiology shows that brushing teeth frequently is linked with lower risks of atrial fibrillation (irregular heartbeat) and heart failure (the heart's ability to pump blood or relax and fill with blood is impaired). ${ }^{1}$

The retrospective cohort study enrolled 161,286 participants of the Korean National Health Insurance System aged 40 to 79 with no history of atrial fibrillation or heart failure. Participants underwent a routine medical examination between 2003 and 2004. Information was collected on height, weight, laboratory tests, illnesses, lifestyle, oral health, and oral hygiene behaviours.

During a median follow-up of 10.5 years, 4,911 (3.0\%) participants developed atrial fibrillation and 7,971 (4.9\%) developed heart failure.
Tooth brushing three or more times a day was associated with a $10 \%$ lower risk of atrial fibrillation and a $12 \%$ lower risk of heart failure during 10.5-year follow up. The findings were independent of a number of factors including age, sex, socioeconomic status, regular exercise, alcohol consumption, body mass index, and comorbidities such as hypertension.

While the study did not investigate mechanisms, one possibility is that frequent tooth brushing reduces bacteria in the subgingival biofilm, thereby preventing translocation to the bloodstream.

Senior author Dr Tae-Jin Song of Ewha Womans University, Seoul, Korea noted that the analysis was limited to one country and as an observational study does not prove causation. But he added: 'We studied a large group over a long period, which adds strength to our findings'.

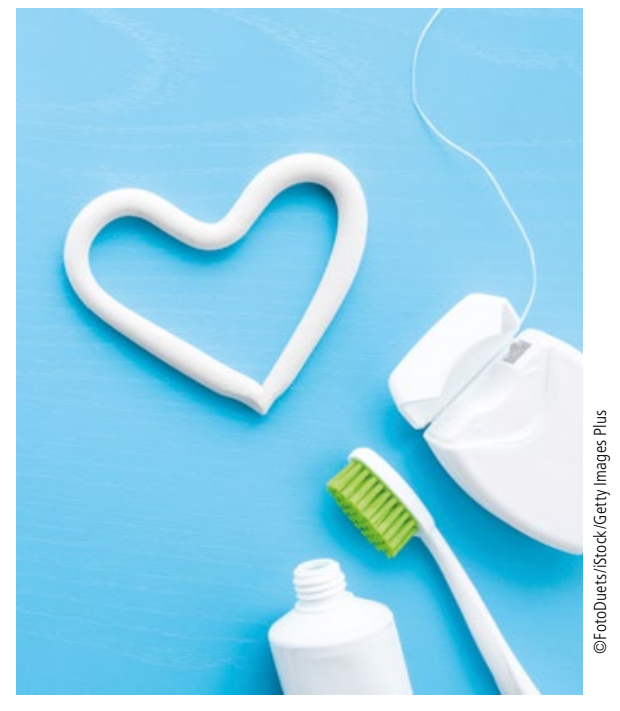

\section{Reference}

1. Chang Y, Woo H G, Park J, Lee J S, Song T J. Improved oral hygiene care is associated with decreased risk of occurrence for atrial fibrillation and heart failure: a nationwide population-based cohort study. Eur J Prev Cardiol 2019; doi: 10.1177/2047487319886018. [Epub ahead of print]. 